\title{
Akathisia — rare cause of psychomotor agitation in patients with traumatic brain injury: Case report and review of literature
}

\author{
Janet E. Wielenga-Boiten, MD; Gerard M. Ribbers, MD, PhD* \\ Rotterdam Neurorehabilitation Research (RoNeRes), Rijndam Rehabilitation Centre, and Department of Rehabilitation \\ Medicine, Erasmus MC, Rotterdam, the Netherlands
}

\begin{abstract}
Akathisia is reported to be one of the most common and disabling side effects of antipsychotics and other drugs. Akathisia is also a rare cause of psychomotor agitation in patients with traumatic brain injury (TBI). In this clinical note, we describe a case report of akathisia in a 34-year-old woman with TBI; review earlier case studies on akathisia in TBI; and discuss the differential diagnosis and its pathophysiology, treatment, and prognosis.
\end{abstract}

Key words: agitation, akathisia, brain injury, case report, delirium, motor restlessness, neuroleptics, psychomotor agitation, rehabilitation, sedatives, traumatic brain injury.

\section{INTRODUCTION}

Akathisia is a rare clinical condition that can occur after traumatic brain injury (TBI). It may cause psychomotor agitation and can easily be overlooked or misinterpreted, for example, as delirium [1]. In this clinical note, we describe a case of akathisia in a 34-year-old woman with TBI. In addition, we review earlier case studies of akathisia in patients with TBI and discuss the differential diagnosis as well as its pathophysiology, treatment, and prognosis.

\section{CASE DESCRIPTION}

A nondisabled 34-year-old woman was involved in a bicycle accident and experienced a TBI. She was admitted to the hospital with a Glasgow Coma Scale (GCS) score of 10. A computed tomography (CT) scan showed a subdural hematoma left frontoparietal, a fracture of the occipital condyle, diffuse axonal injury, and hemorrhage in the globus pallidus and internal capsule of the right hemisphere. After 3 days, she was transferred from the intensive care unit to the neurological ward. Because of agitation, the patient was restrained on the bed or on a wheelchair. She was not prescribed neuroleptics. After 1 week, the patient was discharged to a rehabilitation center. At that time, in the absence of posttraumatic

\footnotetext{
Abbreviations: $\mathrm{CT}=$ computed tomography, GCS = Glasgow Coma Scale, MRI = magnetic resonance imaging, SSRI = selected serotonin reuptake inhibitor, TBI = traumatic brain injury.

*Address all correspondence to Professor Gerard M. Ribbers, MD, PhD; Rijndam Rehabilitation Centre, Westersingel 3003015 LJ Rotterdam, the Netherlands; +31-102412411; fax: +31-10-2412434.
}

Email: g.ribbers@rijndam.nl http://dx.doi.org/10.1682/JRRD.2011.10.0202 
amnesia, she was still agitated and walked around continuously. The patient was not prescribed antidepressants, antiemetics, or calcium-channel blockers. One day after admission to rehabilitation, during the weekend shift and on the assumption that the patient was experiencing delirium, the atypical antipsychotic risperidon was started at a dose of $1 \mathrm{mg}$ twice a day in combination with the benzodiazepine temazepam at a dose of $20 \mathrm{mg}$ ante noctum. The agitated behavior worsened; the patient did not sleep and became exhausted. At reexamination, immediately after the weekend shift, the patient was alert and well oriented. She described a burning sensation spreading down from her abdomen to her legs, followed by an irresistible urge to move. Walking provided relief for only a few minutes. The diagnosis of delirium was rejected, and the antipsychotic medication was stopped. Then, with a diagnosis of akathisia, the $\alpha_{2}$-adrenergic agonist clonidine was prescribed twice a day at a dose of $0.025 \mathrm{mg}$. Within 1 day, the urge to move disappeared completely (Figure) [2]. The patient's heart rate remained stable. After 3 days, the benzodiazepine was also stopped. We attempted to lower the dose of clonidine after 2 weeks, but the symptoms recurred within 24 hours. The initial dose was restarted, and the symptoms disappeared immediately. Two months postinjury, the patient was discharged to outpatient rehabilitation, still using clonidine. During

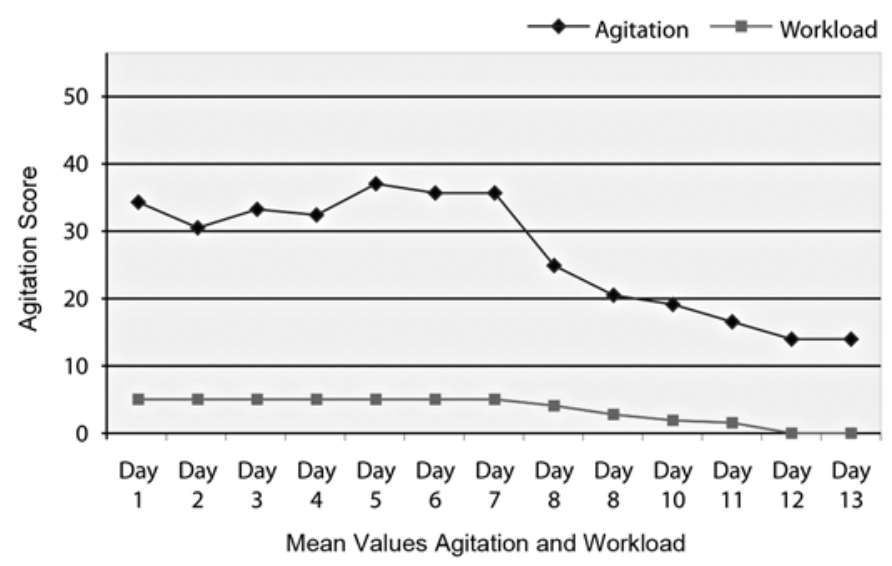

Figure.

Mean values for agitation and workload. Day 1 = patient's first day in rehabilitation center. Figure is translated and adapted version of Agitated Behavior Scale [2], including workload measurement for nursing staff. outpatient rehabilitation, the clonidine was stopped after 3 months, without reemergence of the symptoms.

\section{LITERATURE REVIEW}

Akathisia, derived from the Greek akathemi ("never to sit down"), was first described by Haskovec in 1902 [3]. Patients may typically report a feeling of inner restlessness, causing a compulsion to move. Typically, the patient presents with restless movements such as rocking, walking on the spot when standing, shuffling, or swinging one leg on the other when sitting, in an attempt to relieve the symptoms [4-6]. Akathisia is known as a common and disabling side effect of antipsychotics. Some 20 to 75 percent of patients treated with neuroleptics experience akathisia, especially in the first 3 months of treatment [4,6-8]. If left untreated, akathisia may lead to personal distress, worsening of psychosis, noncompliance, impulsive disruptive behavior, and increased suicide risk [8-10]. It is less known that antiemetics (metoclopramide, prochlorperazine), antidepressants (tricyclics, selective serotonin reuptake inhibitors [SSRIs]), and calcium-channel blockers (cinnarizine, flunarizine, diltiazem) may also cause akathisia [1,9-10]. Akathisia has also been described in patients with Parkinson disease, encephalitis, and subthalamic abscess [1].

We identified four case reports on akathisia in patients with TBI. Stewart described a 61-year-old man who "fell off a ladder, striking his occiput" [11]. The patient was unconsciousness for 4 minutes, and minimal posttraumatic amnesia was reported; the GCS was not described. After the accident (time postonset was not reported), the man had a feeling of motor restlessness, spending the entire day pacing up and down or rocking in a rocking chair. A small right parieto-occipital area of encephalomalacia and much larger bilateral orbitofrontal lesions were visible on CT scanning. The symptoms of restlessness were explained as akathisia. The administration of the benzodiazepine alprazolam at $2 \mathrm{mg}$ per day, later replaced by diazepam at $15 \mathrm{mg}$ per day, resulted in only mild relief of the symptoms. The following month, bromocriptine, a dopamine agonist, was also initiated up to a dose of $7.5 \mathrm{mg}$ per day. This provided complete resolution of the symptoms within several days.

The second case describes a 17-year-old girl who was involved in a motor vehicle accident [12]. A GCS of 4 was reported. During transport to the trauma center, the 
GCS score was 6-7. The girl suffered "bifrontal contusions with associated haemorrhage and sustained multiple orthopaedic and internal injuries.” Because of a sudden collapse in cognitive status 6 days postinjury, a bifrontal debridement and evacuation of the hemorrhages was performed. The dopamine agonist amantadine (dose not reported) was administered to stimulate functional recovery. Because of an increase of agitation, 3 weeks after the injury, haloperidol $(0.5 \mathrm{mg}$ four times a day and $1 \mathrm{mg}$ ante noctum) was initiated (the authors do not report whether the amantadine was stopped before prescribing haloperidol). Haloperidol was discontinued at admission for rehabilitation 6 weeks after the injury. At that time, the girl was alert but agitated, severely restless, and mute. She constantly paced up and down her room and around the hospital unit, repeatedly retracing the same route. The symptoms of restlessness were explained as akathisia. Based on the positive report of Stewart [11], bromocriptine was considered, but after 1 week, her restlessness resolved spontaneously. The patient was discharged home about 4 weeks after admission to the rehabilitation setting. At that time, she had become verbal and well oriented and was off medication.

Hensley and Reeve described the case of a 22-yearold woman involved in a motor vehicle accident sustaining multiple injuries: "bilateral leg and arm fractures, splenic laceration, liver lacerations and closed-head injury (intoxicated, unknown duration of loss of consciousness)" [5]. No information about posttraumatic amnesia or GCS score was reported. The woman had a history of drug and alcohol abuse, posttraumatic stress disorder, depression, and probable anorexia nervosa. At the acute hospital, before transfer to the rehabilitation center, she had gone through alcohol withdrawal, and haloperidol was initiated (dose not reported) because of agitation. The duration of hospital admission was not reported. After arrival at the rehabilitation center, haloperidol was stopped. Lorazepam (dose not reported) was prescribed for the agitation. The SSRI sertraline, $50 \mathrm{mg}$ per day, was added for treatment of depression. After 2 days, the patient became "extremely restless, frequently repositioning herself or requesting that her chair be moved." She reported increased anxiety. Because of the possibility of sertraline-induced anxiety, the sertraline was stopped the next day. However, her symptoms did not improve but worsened. Because of a severe anxiety attack at the site of the magnetic resonance imaging (MRI) scanner, scanning could not be completed. After the diagnosis of depressive disorder contributing to mood lability, the SSRI paroxetine, $10 \mathrm{mg}$ per day, was administered. An abrupt increase in motor restlessness occurred after the first dose of paroxetine. The paroxetine was stopped, and a tricyclic agent was initiated. The symptoms, explained as a result of antidepressant-induced akathisia, did not recur.

Finally, Desai et al. described a 13-year-old boy (weighing $70 \mathrm{~kg}$ ) who "was involved in an unhelmed bicycle crash while riding down a hill, with loss of consciousness for a few seconds" [1]. At the emergency room, he received several doses of various sedatives because he was “confused, agitated, and tearful.” In total, he received $9 \mathrm{mg}$ haloperidol, $6 \mathrm{mg}$ of the short-acting benzodiazepine midazolam, and $1.5 \mathrm{mg}$ hydromorphone (a narcotic analgesic), all intravenously. CT scanning showed no abnormality. The next day he was unable to sleep because of motor restlessness, which caused him to continuously pace around his room in a state of "confusion, marked anxiety and with a sensation of terror." He demonstrated dystonic postures of his left hand and a mild torticollis. CT imaging was repeated, and MRI was also performed, both showing no abnormality. Despite several small (unreported) doses of the benzodiazepine lorazepam, the symptoms worsened that same day. The diagnosis was dystonia and akathisia, both secondary to haloperidol administration. The patient was treated with lorazepam, $1 \mathrm{mg}$ every 8 hours; the selective $\beta_{1}$-blocker metoprolol, 25 mg every 8 hours; and additional diphenhydramine (a sedative with anticholinergic effects), $50 \mathrm{mg}$ as required. The symptoms of akathisia, dystonia, and confusion improved gradually over 4 to 5 days. Discharge to home occurred on hospital day 6. Two weeks after discharge, his symptoms and signs had completely resolved.

\section{DISCUSSION}

It is important to differentiate akathisia from agitation. Agitation after TBI is an excessive global psychomotor activity, inappropriate to the environment, and may be a manifestation of delirium but frequently exists independently of it [13]. It is a complex problem that may hinder rehabilitation and cause distress and even physical harm to the patient and relatives. A widespread sequence of possible diagnoses may underlie psychomotor agitation after TBI, such as anxiety, nonakathisia 
antipsychotic dysphoria, agitation secondary to psychotic symptoms, mania, drug-withdrawal states, organic disorders (e.g., delirium, hypoglycemia, encephalitis lethargica, epilepsy, endocrine dysfunction, pain), agitation related to affective disorders, neurologic disorders (e.g., Parkinson disease, Huntington disease), tardive dyskinesia (commonly coexists with akathisia), myoclonus, acute dystonia, and tremor [7,14-16]. It is not possible to identify one single underlying mechanism of posttraumatic agitation. In the acute posttraumatic phase, agitation may be severe and undifferentiated, whereas in later stages, aggression and episodic dyscontrol may predominate. Different neurochemical axes may be involved, and patterns of agitation may change over time. Neurochemical dysregulations of the serotonergic and dopaminergic or different combinations of neurotransmitter systems may underlie posttraumatic agitation, and many different pharmacologic interventions have been described. Because of methodological shortcomings, effective pharmacological interventions remain unproven [17]. In the case of posttraumatic agitation, nonpharmacologic treatment, such as scheduling, reducing distracters, creating a predictable environment, and sometimes physical restraint, is the first choice. When environmental and behavioral measures are unsuccessful, some form of pharmacologic intervention is necessary. There is no single "magic bullet" to treat posttraumatic agitation. It requires an individual approach, taking into account the phase postonset, the mechanism of the brain injury, and comorbidities.

Our case illustrates that akathisia may cause postacute traumatic agitation to be misinterpreted as delirium. Our patient described a constant sense of motor restlessness without clear reference to cognitive dysfunctions and in the absence of disruptive behavior such as aggression. Akathisia is a nosological entity that should be considered in the agitated TBI patient, requiring a specific treatment. Although akathisia is mostly described as a side effect of neuroleptics (such as haloperidol), it may be related to the location of the brain injury. To our knowledge, our case report is the second one on akathisia caused by TBI, without any relation to the use of antipsychotics.

In the case reports of Desai et al. [1] and Hensley and Reeve [5], previous administration of antipsychotics was reported, and in these cases, there was no evidence for visible intracranial injury. Silver and Yablon reported previous administration of amantadine and haloperidol and bifrontal contusions with associated hemorrhage on
CT imaging [12]. In these cases, it is unclear whether akathisia is related to TBI or to the use of antipsychotics. It is possible that the effect of medication was augmented by the TBI. Teng et al. described a patient with severe TBI with decerebrate posturing [18]; because of significant lower-left leg restlessness, the patient developed ulcers. After several trials with loxapine, haloperidol, propanolol, methylphenidate, diazepam, and paroxetine, the patient finally responded to bupropion [18]. This might be a case of akathisia. However, the cognitive status of this latter patient seems to be much worse than our patient, and she received loxapine and haloperidol over longer periods of time. Both of these latter medications may cause akathisia as a side effect.

In the case described by Stewart, onset of symptoms was not preceded by the use of antipsychotics [11]; CT imaging revealed bifrontal injury. Similar to Stewart's report, in our case, the psychomotor agitation was misinterpreted. Our patient was erroneously diagnosed as having delirium and was prescribed an antipsychotic and a benzodiazepine. However, as our patient was well oriented, capable of reflecting on the situation, and showed no disruptive behavior, the diagnosis of delirium was rejected immediately after the weekend shift. The medication was changed to clonidine. Within 24 hours of starting clonidine, the symptoms that had been present for over 2 weeks had disappeared. Gualtierie also described the treatment of akathisia with clonidine [3]. Stewart effectively treated his patient with bromocriptine [11], and in the case of Silver and Yablon, the symptoms resolved spontaneously [12].

Although there are several hypotheses on the pathophysiology of acute akathisia, not one is completely satisfactory. As stated by Kumar and Sachdev, the most attractive hypothesis is dopamine receptor blockade in the mesocortical and mesolimbic regions of the brain, which leads to decreased dopaminergic activity [9]. However, others state that it is unlikely that a single neurotransmitter hypothesis will explain all the complex features of the disorder. The interaction of several neurotransmitters may be involved, such as dopamine, acetylcholine, gamma-aminobutyric acid, norepinephrine, serotonin, and neuropeptides [1,6-7,9,11,16].

Currently, no consensus exists on the treatment of choice for akathisia. Case reports and small randomized studies have suggested that in addition to $\beta$-adrenergic blockers and dopamine agonists, benzodiazepines and anticholinergic agents may also be effective. Additionally, 
once symptom control is achieved and potentially offending medication is removed, the long-term prognosis is good [1,9,14-15]. If akathisia is misdiagnosed as delirium and treated with neuroleptics, the clinical picture may not improve, challenging the clinician to augment the dose of neuroleptics; the symptoms may then deteriorate.

\section{CONCLUSIONS}

Akathisia is a rare cause of psychomotor agitation in patients with TBI. Its pathophysiology is poorly explained, and no well-accepted treatment algorithm exists. It is essential that this diagnosis is considered in the differential diagnosis of a patient that is agitated following TBI and that potential offending medication is stopped or substituted. Preferably, patients with TBI should not be given sedatives such as antipsychotics, benzodiazepines, and anticholinergics. Therefore, clonidine and bromocriptine seem to be the medications of first choice for TBI patients with motor restlessness that cannot be explained by cognitive disorders.

\section{ACKNOWLEDGMENTS}

\section{Author Contributions:}

Study concept and design: G. M. Ribbers, J. E. Wielenga-Boiten. Acquisition of data: G. M. Ribbers, J. E. Wielenga-Boiten. Analysis and interpretation of data: G. M. Ribbers, J. E. WielengaBoiten.

Drafting of manuscript: G. M. Ribbers, J. E. Wielenga-Boiten. Critical revision of manuscript for important intellectual content: G. M. Ribbers, J. E. Wielenga-Boiten.

Administrative, technical, or material support: G. M. Ribbers, J. E. Wielenga-Boiten.

Study supervision: G. M. Ribbers.

Financial Disclosures: The authors have declared that no competing interests exist.

Funding/Support: This material was unfunded at the time of manuscript preparation.

\section{REFERENCES}

1. Desai A, Nierenberg DW, Duhaime AC. Akathisia after mild traumatic head injury. J Neurosurg Pediatr. 2010;5(5): 460-64. [PMID:20433258] http://dx.doi.org/10.3171/2009.11.PEDS09389

2. Corrigan JD. Development of a scale for assessment of agitation following traumatic brain injury. J Clin Exp Neuro- psychol. 1989;11(2):261-77. [PMID:2925835]

http://dx.doi.org/10.1080/01688638908400888

3. Gualtierie CT. Neuropsychiatry and behavioral pharmacology. New York (NY): Springer-Verlag; 1991. p. 213-16.

4. Ayd FJ. Lexicon of psychiatry, neurology and the neurosciences. 1st ed. Baltimore (MD): William \& Wilkins; 1995. p. 15-16.

5. Hensley PL, Reeve A. A case of antidepressant-induced akathisia in a patient with traumatic brain injury. J Head Trauma Rehabil. 2001;16(3):302-5. [PMID:11346452] http://dx.doi.org/10.1097/00001199-200106000-00009

6. Lima AR, Soares-Weiser K, Bacaltchuk J, Barnes TR. Benzodiazepines for neuroleptic-induced acute akathisia. Cochrane Database Syst Rev. 2002;(1):CD001950. [PMID:11869614]

7. Kane JM, Fleischhacker WW, Hansen L, Perlis R, Pikalov A 3rd, Assunção-Talbott S. Akathisia: an updated review focusing on second-generation antipsychotics. J Clin Psychiatry. 2009;70(5):627-43. [PMID:19389331] http://dx.doi.org/10.4088/JCP.08r04210

8. Brüne M. Acute neuroleptic-induced akathisia in patients with traumatic paraplegia: two case reports. Gen Hosp Psychiatry. 1999;21(5):386-88. [PMID:10572782]

9. Kumar R, Sachdev PS. Akathisia and second-generation antipsychotic drugs. Curr Opin Psychiatry. 2009;22(3): 293-99. [PMID:19378382] http://dx.doi.org/10.1097/YCO.0b013e32832a16da

10. Barnes TR. The Barnes Akathisia Rating Scale—revisited. J Psychopharmacol. 2003;17(4):365-70. [PMID:14870947] http://dx.doi.org/10.1177/0269881103174013

11. Stewart JT. Akathisia following traumatic brain injury: treatment with bromocriptine. J Neurol Neurosurg Psychiatry. 1989;52(10):1200-1201. [PMID:2795050] http://dx.doi.org/10.1136/jnnp.52.10.1200

12. Silver BV, Yablon SA. Akathisia resulting from traumatic brain injury. Brain Inj. 1996;10(8):609-14. [PMID:8836518]

13. Rowland T, DePalma L. Current neuropharmacologic interventions for the management of brain injury agitation. NeuroRehabilitation. 1995;5:219-32.

14. Van Harten PN. Acathisie als bijwerking van geneesmiddelen [Akathisia as side effect of medication]. Ned Tijdschr Geneeskd. 2002;46(3). Dutch.

15. Lombard LA, Zafonte RD. Agitation after traumatic brain injury: considerations and treatment options. Am J Phys Med Rehabil. 2005;84(10):797-812. [PMID:16205436] http://dx.doi.org/10.1097/01.phm.0000179438.22235.08

16. Iqbal N, Lambert T, Masand P. Akathisia: problem of history or concern of today. CNS Spectr. 2007;12(9, Suppl 14):1-13. [PMID:17805218]

17. Levy M, Berson A, Cook T, Bollegala N, Seto E, Tursanski S, Kim J, Sockalingam S, Rajput A, Krishnadev N, Feng C, 
Bhalerao S. Treatment of agitation following traumatic brain injury: a review of the literature. NeuroRehabilitation. 2005;20(4):279-306. [PMID:16403996]

18. Teng CJ, Bhalerao S, Lee Z, Farber J, Morris H, Foran T, Tucker W. The use of bupropion in the treatment of restlessness after a traumatic brain injury. Brain Inj. 2001; 15(5):463-67. [PMID:11350660]

Submitted for publication October 27, 2011. Accepted in revised form February 23, 2012.
This article and any supplemental material should be cited as follows:

Wielenga-Boiten JE, Ribbers GM. Akathisia-rare cause of psychomotor agitation in patients with traumatic brain injury: Case report and review of literature. J Rehabil Res Dev. 2012;49(9):1349-54. http://dx.doi.org/10.1682/JRRD.2011.10.0202

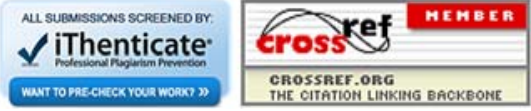

